\title{
DETECTION OF BIAS IN THE BACKGROUND OF VIALS USED FOR RADIOCARBON DATING
}

\author{
JANET AMBERS, MORVEN LEESE, and SHERIDAN BOWMAN
}

Research Laboratory, The British Museum, London WC1 B 3DG

ABSTRACT. Inter-vial variation in background of the glass vials used in liquid scintillation counting can introduce appreciable errors into ${ }^{14} \mathrm{C}$ measurements. Our aim was to measure the background in each of 50 glass vials, under the same conditions as far as possible, in order to find a self-consistent set for use in ${ }^{14} \mathrm{C}$ dating. The criteria, statistical tests, and possible errors introduced by not making such checks are discussed.

\section{INTRODUCTION}

Vials of low-potassium glass are frequently used for ${ }^{14} \mathrm{C}$ dating by liquid scintillation counting. That such vials can have different backgrounds was recorded as early as 1965 (Garfinkel et al, 1965; Painter, 1974, p 435). Clearly if unknown and background samples are counted in vials having inherently different background levels, a systematic error in the age determination of the unknown sample will result. Pearson (1979) found a large spread of background measurements in preliminary trials with 500 vials and overcame the problem by selection by weight, masking of the upper part of each vial, and extreme precision in weighing out of samples. Given the errors that can be introduced by the use of vials with differing backgrounds, it would seem worthwhile to take a batch of vials and select a consistent sub-set which can then be continually re-used for routine dating, under the normal conditions used in non-high-precision laboratories; this avoids the necessity for an additional correction factor specific to each vial. We have found no references in the literature that detail how to reject vials from a batch on the basis of background. This paper suggests criteria and procedures by which the difference (or bias as we have termed it) between the background of a vial and some predetermined target value may be detected.

\section{EXPERIMENTAL METHOD}

Fifty $20 \mathrm{ml}$ low-potassium glass vials (Packard Instruments Ltd, England) were tested in 3 liquid scintillation counters: a Packard Tri-carb model 3003 (counter 1), a Packard Tri-carb model 3255 (counter 2), and a I.KB Rackbeta model 1217 fitted with the 'Kangaroo' ${ }^{14} \mathrm{C}$ package (counter 3). Under normal circumstances the background level in the 2 Packard counters is ca 8 and 10 counts per minute $(\mathrm{cpm})$, respectively, and that in the $\mathrm{LKB}$ counter, ca $4 \mathrm{cpm}$. All 3 machines run at slightly different efficiencies and count rates from any 1 machine cannot be directly compared with those from another. At any one time approximately one third of the vials were in a given counter. A mixture of $5.5 \mathrm{ml}$ 'analar' benzene (supplied by $\mathrm{BDH}$, England), which was known to have similar quench characteristics to the benzene routinely produced from samples in the laboratory, and $9.5 \mathrm{ml}$ of a scintillator solution of PPO (2, 5-diphenyloxazole) in scintillation grade toluene (both supplied by $\mathrm{BDH}$ ) was added to each vial. The cardboard and tin seals supplied with the vials were used. The liquid levels in the viais were monitored to ensure that apparent differences in background were not due 
to liquid loss, and the weights of each vial, vial cap, benzene and total liquid contents were noted. No vial or photomultiplier tube masking was used. The samples were counted in trains with a 50 -minute counting period. From experience of routine measurement, no change in quench or counting efficiency was expected over the period of the experiment. The total count time needed to produce statistically acceptable figures will be discussed below. Two sets of experimental measurements, A and B, were produced and used to define tests for the rejection of vials, given certain criteria.

\section{THST CONDITIONS}

\section{Criteria Used} follows:

The criteria needed for the application of the statistical tests were as

1) Target value (T) for the background in cpm. A central value such as the mean of the vials will lead to the retention of a large number of them. Any value can be chosen, eg, consistency may be ensured with an existing set of vials by using a previously determined target value.

2) Allowable rejection rate $(\mathbb{R})$. This is the significance level, or the 'false positive' percentage of unbiased vials that may have to be sacrificed unnecessarily to pick up any real biases. We decided that $20 \%$ of the unbiased vials were expendable over the two experiments, $10 \%$ in each.

3) Difference (I)) from the target that it is essential to identify and hence eliminate. From consideration of the age equation it is clear that if systematic differences exist, the resultant error on the age can be substantial and, even for relatively active samples, may be larger than that based on counting statistics, which is frequently the only error quoted on a measurement. If $\mathrm{I})$ were $0.5 \mathrm{cpm}$, eg, the additional systematic error on a $5.5 \mathrm{ml} \mathrm{sam-}$ ple only one half-life old would be $3.4 \%$ (given a counting efficiency of ca $70 \%)$ and, under the same conditions, if $\mathrm{D}$ were $0.25 \mathrm{cpm}$, the resultant crror on the same sample would be $1.7 \%$. It would have been ideal to eliminate biases as small as $0.1 \mathrm{cpm}$, but that would have involved an inordinately

TABLE 1

Number of cycles (and days) needed to distinguish vials with background at least I) (cpm) different from a target figure

\begin{tabular}{cccc}
\hline 1) & $\begin{array}{c}\text { Allowable } \\
\text { rejection } \\
\text { rate } \\
(\mathrm{pm})\end{array}$ & $\mathrm{R}(\%)$ & \multicolumn{2}{c}{$\begin{array}{c}\text { Number of cycles to ensure } \\
\text { 1) is detected with probability P }\end{array}$} \\
\cline { 2 - 4 } 0.25 & 20 & $90 \%$ & $95 \%$ \\
\hline .1 & 10 & $21(36)^{* *}$ & $33(57)$ \\
0 & 30 & $117(203)$ & $185(321)$ \\
\hline
\end{tabular}

* Number, $\mathrm{n}$ of $5($ )-minute counts needed on cach vial where the assumed background is $8($ pm. For given $\mathrm{D}), \mathrm{R}$ and $\mathrm{P}, \mathrm{n}$ is proportional to $\mathrm{B}$, and inversely proportional to the cycle time $m$.

** Figures in parentheses are the number of days required to count 50 vials. 
long experimental time (see below and Table 1). We compromised on a value of $0.25 \mathrm{cpm}$.

Using these criteria, a standard ' $t$ ' test was used to reject vials deviating significantly from the target. A vial was rejected if:

$$
|\mathrm{M}-\mathrm{T}| / \mathrm{s}>\mathrm{t}_{\mathrm{R}}
$$

where $\mathrm{M}$ is the measured mean background for that vial, $\mathrm{s}$ is the measurement error on $M$, and $t_{R}$ the ' $t$ '-value corresponding to a significance level of $\mathrm{R} \%$ (the allowable rejection rate for good vials) with the appropriate degrees of freedom for s. When the number of cycles from which the mean is computed is large ( $\mathrm{say}>100), \mathrm{t}_{\mathrm{R}}$ can be approximated by $\mathrm{Z}_{\mathrm{R}}$, a Normal deviate, as tabulated, eg, in Snedecor and Cochran (1967). As an instance, the value of $Z_{20 \%}$ is 1.28 .

\section{Counting Time Required to Detect Given Bias}

Assuming all the vials are to be counted in a single counter, we can now consider the time required to ensure that the test will reject vials with bias at least as high as $\mathrm{I}$ with probability $\mathrm{P} \%$. Following the procedures of Snedecor and Cochran (1967) P can be found approximately from

$$
\mathrm{Z}_{2(100-\mathrm{P}) \%}=\mathrm{D} / \mathrm{s}-\mathrm{Z}_{\mathrm{R} \%}
$$

where $Z_{2(100-P) \%)}$ and $Z_{R}$ are the values of a Normal deviate corresponding to the $2(100-\mathrm{P}) \%$ and the $\mathrm{R} \%$ point, respectively; $\mathrm{s}$ is the standard error of the difference of the vial mean from the target $\mathrm{T}$. Since $\mathrm{T}$ is a constant, $\mathrm{s}$ is simply equal to the standard error of the mean.

The approximate error can be predicted using counting statistics. If the average background is $\mathrm{B} \mathrm{cpm}$, the standard error on a measurement over a single cycle of $\mathrm{m}$ minutes will be $\mathrm{s}=\sqrt{\mathrm{B} / \mathrm{m}}$ and the error in the mean of $n$ such values will be $\sqrt{B /(m n)}$. Substituting for $s$ in (2) and solving for $n$ gives the number of cycles required:

$$
\mathrm{n}=\left(\mathrm{Z}_{2(100-\mathrm{P}) \%}+\mathrm{Z}_{\mathrm{R} \%}\right)^{2}(\mathrm{~B} / \mathrm{m}) / \mathrm{D}^{2}
$$

The necessary counting time in a single counter is given in Table 1, for various combinations of test criteria. Using this table, or alternatively substituting appropriate values into Equation (3), looking up Normal tables for $Z$, we can compute the counting time that will be sufficient to ensure the elimination of biases inconsistent with any given dating requirements, without undue wastage of good vials.

\section{THE EXPERIMENTS}

Since we wanted to keep the counting time needed to make this selection to a minimum, we split the batch of 50 vials into 3 groups of approximately equal size and counted each group separately in 1 of the 3 counters (Experiment B). The groups were counted for 37 cycles in Counters 1 and 2 and 22 cycles in Counter 3 . A further set of measurements was made and each vial counted for eight 50-minute periods in each of the 3 counters (Experiment A). This was arranged by swapping the 3 batches of vials from 
one counter to another at intervals of 8 cycles, thus eliminating inter-counter differences. The results of Experiment $B$ were used primarily as a backup to Experiment $A$, and they also enabled the detection of any gross outliers before the main analysis of the data. For this reason the experiments were labeled in logical, rather than chronological, order.

\section{Analysis of the Data}

The data produced by Experiment A were subjected to an analysis of variance; this 'stripped off' the variations due to differences between counters and time periods, leaving only the measurement error which could be used for inter-vial comparison. Applying the criteria and test discussed above, 9 vials were rejected as differing from the selected target value (7.16cpm-the grand mean of all the measurements) with an allowable rejection rate of $10 \%$. The probability that this identified biases of at least $0.25 \mathrm{cpm}$ was ca $92 \%$. The number rejected constitutes $18 \%$ of the vials. The fact that this is greater than the $10 \%$ allowed rejection rate demonstrates that differences in background $>0.25 \mathrm{cpm}$ do exist under these conditions. The normalized mean counts for the vials are shown in Figure 1, together with the target value and acceptable limits as defined by the test.

In Experiment $\mathrm{B}$ we used a target value equivalent to that used in Experiment $\mathrm{A}$ and the same $\mathrm{D}$ and $\mathrm{R}$ values. This time 10 vials were rejected, $20 \%$ of the total. In this case the confidence was ca $97-99 \%$. Of these 10 vials, 4 were also rejected by Experiment $\mathrm{A}$.

We decided to reject vials which failed either of the two tests, giving an overall confidence of $>99 \%$ that all vials with a bias $>0.25 \mathrm{cpm}$ had been detected, and entailing a risk that ca $20 \%$ of the unbiased vials had been rejected. In all, 16 vials from the original 50 were rejected, $32 \%$ of the total.

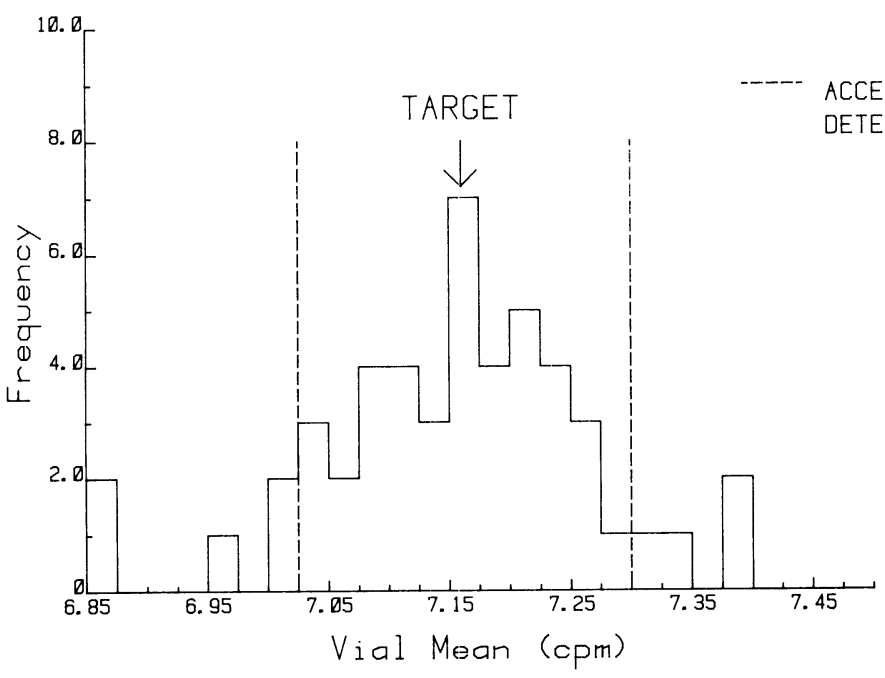

Fig 1. Vial means-Experiment A 


\section{DISCUSSION}

It is clear that bias in the background of glass vials will introduce appreciably large errors to ${ }^{14} \mathrm{C}$ dates produced by liquid scintillation counting. These experiments show that such biases exist and need to be climinated. In practical terms it will always be necessary to reject and remove some vials that do not have true bias to ensure that all bias is detected.

There is very little doubt that the four vials rejected by both tests differ significantly from the target. Envisaging the worst possible case, in which we were to unwittingly use these vials, we could encounter a bias of ca $0.5 \mathrm{cpm}$ in $7 \mathrm{cpm}$, or the equivalent of an error of ca $4.5 \%$ on a date of one half-life and significantly more for older samples.

These experiments were conducted to produce vials for use under our normal counting conditions; no account was taken of possible orientation effects as reported by Garfinkel et al (1965) since the sample conveyer system in the scintillation counters causes rotation of the vials between counting periods which should average out any such differences.

It has been suggested that bias in the background of a set of counting vials can be avoided by selecting vials of closely similar weights. The weight distribution of vials (without caps) rejected by these tests (Fig 2) does not support this contention. Three of the 4 vials rejected by both tests lie in the central part of the distribution, very close to the modal weight.

No extra precautions were taken with vial filling and there was a range of content weights. All but one of the vials had contents weighting between 13.0 and $13.3 \mathrm{~g}$. The background of the single vial with contents weighing $0.5 \mathrm{~g}$ less than the average was ca $0.3 \mathrm{cpm}$ lower than average, a reduction of the order suggested by the corrections of Pearson et al (1977). However no

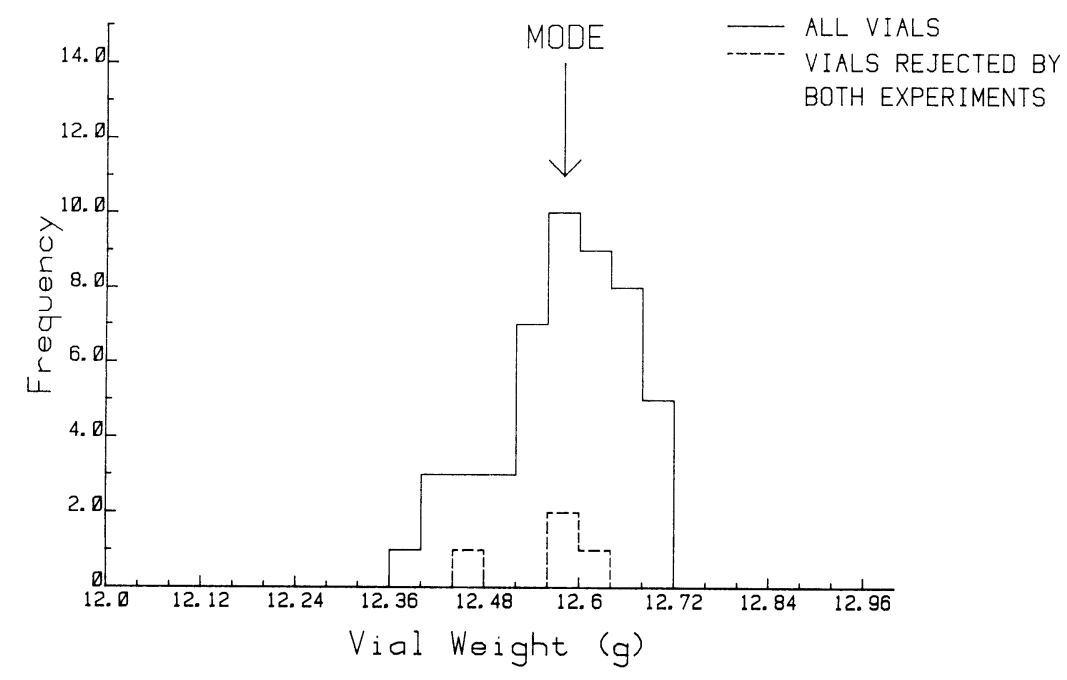

Fig 2. Distribution of vial weights 
correlation was observed between the weight of the vial contents and rejection by these tests. We conclude that the majority of the differences observed are due to properties of the vials themselves.

In retrospect, we realized that the method of Experiment $B$ was not ideal and recommend that only Experiment A be used for future work, the appropriate number of cycles being determined by Equation 3 and Table 1, and checks made beforehand that the weight range for the contents lies within acceptable limits.

\section{REFERENCES}

Garfinkel, S B, Mann, W B, Medlock, R W and Yura, O, 1965, The calibration of the National Bureau of Standards' tritiated-toluene standard of radioactivity: Internatl Jour Applied Radiation Isotopes, v 16, p 27-34.

Painter, K, 1974, Choice of counting vial for liquid scintillation: A review, in Stanley, P E and Scroggins, B A, eds, Liquid scintillation counting, recent developments: Internatl symposium on liquid scintillation counting, Sydney, Australia, Aug 20-22, 1973, Proc: New York, Academic Press, p 431-452.

Pearson, G, 1979, Precise ${ }^{14} \mathrm{C}$ measurement by liquid scintillation counting: Radiocarbon, $v$ $21, \mathrm{p} 1-21$

Pearson, G, Pilcher, J, Baillie, M and Hillam, J, 1977, Asbolute radiocarbon dating using a low altitude European tree-ring calibration: Nature, v 270, p 25-28.

Snedecor, G and Cochran, W, 1967, Statistical methods, 6th ed: Iowa, Iowa State Univ Press. 